The first sum in the derivative can be evaluated by the same process used to find $f(\mu)$ where the $B_{r, q}$ now replaces the $c_{r, q}$. The second sum may be evaluated by an obvious application of the algorithm given by Smith.

5. Advantages and Test Case. A simple computation shows that for $n>3$ the new algorithm saves about $3 n$ multiplications and additions when computing $f(\mu)$ and about $n+\sum_{k=3}^{n} k$ multiplications and $\sum_{k=1}^{n} k$ additions when computing $f^{\prime}(\mu)$.

This algorithm has been used successfully in a Fortran application on an IBM 7094 using Legendre Polynomials. We found computation time to be reduced by approximately $25 \%$ and accuracy increased by .5 to one order of magnitude.

6. Acknowledgments. I wish to thank W. E. Daniels for helpful suggestions and G. O'Brien for carrying out the computations on the IBM 7094.

Goddard Space Flight Center

Greenbelt, Maryland 20771

1. F. J. Sмгтн, "An algorithm for summing orthogonal polynomial series and their derivatives with applications to curve-fitting and interpolation," Math. Comp., v. 19, 1965, pp. 33-36. MR 30 *2664.

2. C. W. Clenshaw, "A note on the summation of Chebyshev series," MTAC, v. 9, 1955, pp. 118-120. MR 17, 194.

3. C. W. Clenshaw, G. F. Miller \& M. Woodger, "Algorithms for special functions. I," Handbook Series Special Functions, N'umer. Math., v. 4, 1962/63, pp. 403-419. MR 27 \#4346.

4. J. H. Wilkinson, Rounding Errors in Algebraic Processes, Prentice-Hall, Englewood Cliffs, N. J., 1963. MR 28 * 4661.

\title{
A Note on the Summation of the Generalised Hypergeometric Functions
}

\section{By Arun Verma}

1. Introduction. In 1962 MacRobert and Ragab [2], obtained the sum of an infinite series of $E$-functions as a product of gamma functions. Recently, the author [4] has extended the result to obtain the sum of a series of $G$-functions as a product of gamma functions. Carlitz [1], in a recent paper obtained some results of a different nature. He obtained the sum of a finite number of terms of hypergeometric series as a product of gamma functions. Slater [3] has also given the sum of a generalised hypergeometric function in terms of elementary functions under a set of conditions.

In this note the sum of certain generalised hypergeometric functions, bilateral hypergeometric functions, generalised basic hypergeometric functions and the generalised basic bilateral hypergeometric functions are deduced.

2. Notation. Let

then

$$
[a]_{n}=a[a+1][a+2] \cdots[a+n-1] ; \quad[a]_{0}=1
$$

$$
{ }_{r} F_{s}\left[\begin{array}{c}
\left(a_{r}\right) ; z \\
\left(b_{s}\right)
\end{array}\right]_{N}=\sum_{n=0}^{N} \frac{\left[\left(a_{r}\right)\right]_{n}}{[1]_{n}\left[\left(b_{s}\right)\right]_{n}} z^{n} .
$$

Received August 26, 1966. 
As $N \rightarrow \infty$, the left-hand side tends to the generalised hypergeometric function ${ }_{r} F_{s}\left[\left(a_{r}\right) ;\left(b_{s}\right) ; z\right]$ and has a meaning when $r \leqq s+1$, where $\left(a_{r, n}\right)$ means the sequence of parameters $a_{r}, a_{r+1}, \cdots, a_{n}$. Further we define

$$
{ }_{r} H_{r}\left[\begin{array}{c}
\left(a_{r}\right) ; z \\
\left(b_{r}\right)
\end{array}\right]_{N}^{M}=\sum_{n=-M-1}^{N} \frac{\left[\left(a_{r}\right)\right]_{n}}{\left[\left(b_{r}\right)\right]_{n}} z^{n},
$$

and when $M$ and $N \rightarrow \infty$, they will be dropped and the left-hand side becomes the generalised bilateral hypergeometric function and has a meaning when $|z|=1$, and $\operatorname{Rl}\left[\sum\left(b_{r}\right)-\sum\left(a_{r}\right)\right]>0$ if $z=-1$ or $\mathrm{Rl}\left[\sum\left(b_{r}\right)-\sum\left(a_{r}\right)\right]>1$ if $z=1$.

Furthermore, if

$$
\left[q^{a} ; n\right]=\left[1-q^{a}\right]\left[1-q^{a+1}\right] \cdots\left[1-q^{a+n-1}\right] ; \quad\left[q^{a} ; 0\right]=1, \quad|q|<1,
$$

then

$$
{ }_{r} \Phi_{s}\left[\begin{array}{c}
q^{\left(a_{r}\right)} ; z \\
q^{\left(b_{s}\right)}
\end{array}\right]_{N}=\sum_{n=0}^{N} \frac{\left[q^{\left(a_{r}\right)} ; n\right]}{[q ; n]\left[q^{\left(b_{s}\right)} ; n\right]} z^{n}
$$

and as $N \rightarrow \infty$, the left-hand side tends to the generalised basic hypergeometric function ${ }_{r} \phi_{s}\left[q^{\left(a_{r}\right)} ; q^{\left(b_{s}\right)} ; z\right]$ and has a meaning when $|z|<1$.

Lastly, we define

$$
{ }_{r} \Psi_{r}\left[\begin{array}{c}
q^{\left(a_{r}\right)} ; z \\
q^{\left(b_{r}\right)}
\end{array}\right]_{N}^{M}=\sum_{n=-M-1}^{N} \frac{\left[q^{\left(a_{r}\right)} ; n\right]}{\left[q^{\left(b_{r}\right)} ; n\right]} z^{n}
$$

and as $M$ and $N \rightarrow \infty$, the left-hand side tends to become the generalised basic bilateral hypergeometric function ${ }_{r} \Psi_{r}\left[q^{\left(a_{r}\right)} ; q^{\left(b_{s}\right)} ; z\right]$ and has a meaning only if $\left|q^{\Sigma\left(b_{r}\right)-\Sigma\left(a_{r}\right)}\right|<|z|<1$.

3. Slater [3] has shown that

$$
{ }_{A+1} F_{A}\left[\begin{array}{c}
\left(a_{0, A}\right) ; 1 \\
1+\left(b_{A}\right)
\end{array}\right]_{N}=\frac{\left[1+\left(a_{0, A}\right)\right]_{N}}{[1]_{N}\left[1+\left(b_{A}\right)\right]_{N}}
$$

provided

$$
S_{r}(a)=S_{r}(b) ; \quad r=1,2, \cdots, A,
$$

where $S_{r}(a)$ or $S_{r}(b)$ means a sum of all possible combinations of $r$ parameters taken at a time from the parameters $a_{0}, a_{1}, a_{2}, \cdots, a_{A}$ or $b_{1}, b_{2}, b_{3}, \cdots, b_{A}$ respectively.

It was, however, indicated by Carlitz [1] that if we assume $S_{r}(a)=S_{r}(b)$ for $r=1,2, \cdots, A-1$ only, even then the sum of the first $(N+1)$ terms of the truncated generalised hypergeometric function can be found. In fact, we can deduce that:

$$
{ }_{A+2} F_{A+1}\left[\begin{array}{c}
1+\frac{S_{A+1}(a)}{S_{A}(a)-S_{A}(b)},\left(a_{0, A}\right) ; 1 \\
S_{A+1}(a) /\left[S_{A}(a)-S_{A}(b)\right], 1+\left(b_{A}\right)
\end{array}\right]_{N}=\frac{\left[1+\left(a_{0, A}\right)\right]_{N}}{[1]_{N}\left[1+\left(b_{A}\right)\right]_{N}},
$$

provided $S_{r}(a)=S_{r}(b) ; r=1,2, \cdots, A-1$. 
But since

$$
\begin{aligned}
{ }_{A} H_{A}\left[\begin{array}{c}
\left(a_{A}\right) ; \\
1+\left(b_{A}\right)
\end{array}\right]_{N}^{M}=\sum_{n=-M-1}^{N} \frac{\left[\left(a_{A}\right)\right]_{n}}{\left[1+\left(b_{A}\right)\right]_{n}} \\
\qquad{ }_{A+1} F_{A}\left[\begin{array}{c}
1,\left(a_{A}\right) ; 1 \\
1+\left(b_{A}\right)
\end{array}\right]_{N}+\frac{\left[\left(b_{A}\right)\right]}{\left[\left(a_{A}\right)-1\right]}{ }^{A+1} F_{A}\left[\begin{array}{c}
1,1-\left(b_{A}\right) ; 1 \\
2-\left(a_{A}\right)
\end{array}\right]_{M}
\end{aligned}
$$

summing both the series on the right-hand side of (3.4) by (3.1) we get

$$
\begin{aligned}
{ }_{A} H_{A}\left[\begin{array}{c}
\left(a_{A}\right) ; \\
1+\left(b_{A}\right)
\end{array}\right]_{N}^{M}=\frac{[1+N]\left[1+\left(a_{A}\right)\right]_{N}}{\left[1+\left(b_{A}\right)\right]_{N}} \\
+\frac{(-)^{A}[M+1]\left[\left(b_{A}\right)\right]\left[2-\left(b_{A}\right)\right]_{M}}{\left[1-\left(a_{A}\right)\right]_{M+1}}
\end{aligned}
$$

provided

$$
\begin{aligned}
& S_{r}(a)=S_{r}(b) ; \quad r=1,2, \cdots, A, \\
& S_{r}{ }^{\prime}(a)=S_{r}{ }^{\prime}(b) ; \quad r=1,2, \cdots, A,
\end{aligned}
$$

where $S_{i}(a), S_{i}(b), S_{i}{ }^{\prime}(a)$ and $S_{i}{ }^{\prime}(b)$ stand for the sum of all possible combinations of $i$ factors taken at a time from the sequence of numbers $1, a_{1}, a_{2}, \cdots, a_{A}$; $b_{1}, b_{2}, \cdots, b_{A} ; 1,1-b_{1}, 1-b_{2}, \cdots, 1-\left(b_{A}\right)$ and $1-a_{1}, 1-a_{2}, \cdots, 1-a_{A}$ respectively.

Whereas, if we assume the conditions

and

$$
S_{r}(a)=S_{r}(b) ; \quad r=1,2, \cdots, A-1,
$$

$$
S_{r}^{\prime}(b)=S_{r}^{\prime}(a) ; \quad r=1,2, \cdots, A-1,
$$

we get the following summation theorem

$$
\begin{aligned}
{ }_{A+1} H_{A+1}\left[\begin{array}{c}
1+S_{A+1}(a) /\left[S_{A}(a)-S_{A}(b)\right],\left(a_{A}\right) ; \\
S_{A+1}(a) /\left[S_{A}(a)-S_{A}(b)\right], 1+\left(b_{A}\right)
\end{array}\right]_{N}^{M}=\frac{[1+N]\left[1+\left(a_{A}\right)\right]_{N}}{\left[1+\left(b_{A}\right)\right]_{N}} \\
+\frac{(-)^{A-1}[1+M]\left[\left(b_{A}\right)\right]\left[S_{A}(a)-S_{A}(b)-S_{A+1}(a)\right]\left[2-\left(b_{A}\right)\right]_{M}}{S_{A+1}(a)\left[1-\left(a_{A}\right)\right]_{M+1}} .
\end{aligned}
$$

4. In this section we deduce the basic analogues of the results deduced in the last section.

Since

$$
\begin{aligned}
{ }_{A+1} \Phi_{A}\left[\begin{array}{c}
q^{\left(a_{0, A}\right)} ; z \\
\left.q^{1+\left(b_{A}\right)}\right]_{N}
\end{array}\right. & =\sum_{n=0}^{N} \frac{\left[q^{\left(a_{0, A}\right)} ; n\right]}{[q ; n]\left[q^{1+\left(b_{A}\right)} ; n\right]} z^{n} \\
& =\sum_{n=0}^{N} \alpha_{n} \quad(\text { say }),
\end{aligned}
$$

setting

we have

$$
\vartheta^{n}=\left(1-q\left[{ }^{\left(a_{0, A}\right)+n}\right] \alpha_{n},\right.
$$

$$
\begin{aligned}
\vartheta_{n} & -\vartheta_{n-1} \\
& =\alpha_{n}\left[\sum_{r=0}^{A+1}(-)^{r} S_{r}(a) q^{n r}-\frac{1}{r} \sum_{r=0}^{A+1}(-)^{r} S_{r}(b) q^{n r}+\frac{1}{z} \sum_{r=0}^{A}(-)^{r} S_{r}(b) q^{n(r+1)}\right] .
\end{aligned}
$$

where $S_{r}(a), S_{r}(b)$ means the sum of all possible combinations of $r$ parameters taken 
at a time from the sequence of numbers $q^{a_{0}}, q^{a_{1}}, q^{a_{2}}, \cdots, q^{a_{A}}$ and $q^{b_{1}}, q^{b_{2}}, \cdots, q^{b_{A}}$, respectively. Since there are only $A$ numbers of $b$ parameters, obviously $S_{A+1}(b)=0$.

A little simplification of (4.1) gives that

$$
\vartheta_{n}-\vartheta_{n-1}=\alpha_{n}\left[\sum_{r=0}^{A+1}\left\{S_{r}(a)-\frac{1}{z} S_{r}(b)-\frac{1}{z} S_{r-1}(b)\right\}(-)^{r} q^{n r}\right],
$$

where $S_{-1}(b)=0$.

Now if we assume that the coefficients of all the powers of $q^{n}$ vanish, i.e.

$$
z S_{r}(a)-S_{r}(b)-S_{r-1}(b)=0 \text { for } r=1,2, \cdots, A+1,
$$

we have from $(4.2), \vartheta_{n}-\vartheta_{n-1}=[1-1 / z] \alpha_{n}$.

Giving $n$ the values $0,1,2, \cdots, N$ and adding up, we get

$$
{ }_{A+1} \Phi_{\boldsymbol{A}}\left[\begin{array}{c}
q^{\left(a_{0, A}\right)} ; z \\
q^{1+\left(b_{A}\right)}
\end{array}\right]_{N}=\frac{\left[q^{\left(a_{0, A}\right)} ; N+1\right]}{[q ; N]\left[q^{1+\left(b_{A}\right)} ; N\right]} \cdot \frac{z^{N+1}}{z-1} .
$$

Therefore, (4.4) gives the sum of first $(N+1)$ terms of the generalised basic hypergeometric series, with argument $z$, under the set of conditions (4.3).

On the other hand, if we assume the conditions

$$
z S_{r}(a)-S_{r}(b)-S_{r-1}(b)=0 \text { for } r=2,3, \cdots, A+1,
$$

only, we get the basic analogue of (3.3) in the form:

$$
\begin{gathered}
{ }_{A+2} \Phi_{A+1}\left[\begin{array}{c}
q^{\left(a_{0, A}\right)}, \frac{q\left(z S_{1}(a)-S_{1}(b)-1\right)}{z-1} ; z \\
q^{1+\left(b_{A}\right)}, \frac{z S_{1}(a)-S_{1}(b)-1}{(z-1)}
\end{array}\right]_{N} \\
=\frac{\left[q^{\left(a_{0, A}\right)} ; N+1\right] q z^{N+1}}{\left[(z-1)-z S_{1}(a)+S_{1}(b)+1\right][q ; N]} \times \frac{1}{\left[q^{1+\left(b_{A}\right)} ; N\right]} .
\end{gathered}
$$

It may be noted that if we take $r=1,3,4, \cdots, A+1,(r \neq 2)$ we get still another sum of $N+1$ terms of the basic hypergeometric function ${ }_{A+3} \Phi_{A+2}(z)$.

To find the sum of the $M+N+1$ terms of the basic bilateral hypergeometric series

$$
{ }_{r} \Psi_{r}\left[\begin{array}{c}
q^{\left(a_{r}\right)} ; z \\
q^{\left(b_{r}\right)}
\end{array}\right]_{N}^{M}
$$

we know that

$$
\left.\begin{array}{rl}
{ }_{r} \Psi_{r}\left[\begin{array}{c}
q^{\left(a_{r}\right)} ; z \\
q^{\left(b_{r}\right)+1}
\end{array}\right]_{N}^{M}={ }_{r+1} \Phi_{r}\left[\begin{array}{c}
q, q^{\left(a_{r}\right)} ; z \\
q^{\left(b_{r}\right)+1}
\end{array}\right]_{N} \\
+\frac{\left[1-q^{-\left(b_{r}\right)}\right] q^{r+\Sigma\left(b_{r}\right)-\Sigma\left(a_{r}\right)}}{z\left[1-q^{1-\left(a_{r}\right)}\right]}{ }_{+1} \Phi_{r}\left[q, q^{1-\left(b_{r}\right)} ; \frac{q^{r+\Sigma\left(b_{r}\right)-\Sigma\left(a_{r}\right)}}{z}\right] . \\
q^{2-\left(a_{r}\right)}
\end{array}\right] .
$$

Now if we assume that

$$
\begin{array}{r}
\text { (a) } z S_{k}(a)-S_{k}(b)-S_{k-1}(b)=0 \text { for } k=1,2, \cdots, r+1 \\
\text { (b) } S_{t}^{\prime}(a) \cdot q^{r+\Sigma\left(b_{r}\right)-\Sigma\left(a_{r}\right)}-z\left[S_{t}^{\prime}(b)-S_{t-1}^{\prime}(b)\right]=0 \\
\text { for } t=1,2, \cdots, r+1,
\end{array}
$$


where $S_{r}(a), S_{r}(b), S_{r}^{\prime}(a)$ and $S_{r}^{\prime}(b)$ stand for the sum of all possible combinations of $r$ factors taken at a time from the sequence of numbers $q, q^{a_{1}}, q^{a_{2}}, \ldots, g^{a_{r}}$; $q^{b_{1}}, q^{b_{2}}, \cdots, q^{b_{r}} ; q, q^{1-b_{1}}, q^{1-b_{2}}, \cdots, q^{1-b_{r}}$ and $q^{1-a_{1}}, q^{1-a_{2}}, \cdots, q^{1-a_{r}}$ respectively, we can sum up both the series on the right-hand side of (4.6) and obtain

$$
\begin{aligned}
{ }_{r} \Psi_{r}\left[\begin{array}{l}
q^{\left(a_{r}\right)} ; z \\
q^{1+\left(b_{r}\right)}
\end{array}\right]_{N}^{M}=\frac{\left[1-q^{N+1}\right]\left[q^{\left(a_{r}\right)} ; N+1\right]}{[z-1]\left[q^{1+\left(b_{r}\right)} ; N\right]} \\
+\frac{\left[1-q^{M+1}\right]\left[q^{-\left(b_{r}\right)} ; M+2\right]}{\left[q^{r+\Sigma\left(b_{r}\right)-\Sigma\left(a_{r}\right)}-z\right]} \times \frac{q^{(M+2)\left[r+\Sigma\left(b_{r}\right)-\Sigma\left(a_{r}\right)\right]}}{\left[q^{1-\left(a_{r}\right)} ; M+1\right] z^{M+1}}
\end{aligned}
$$

under the set of conditions (4.7).

On the other hand, if in the set of conditions (7a) and (7b) we assume all the conditions except those corresponding to $k=1$ and $t=1$ we can still find the sum of $M+N+1$ terms of a bilateral hypergeometric function by using the result (5).

Department of Mathematics

Lucknow University

Lucknow, India

1. L. Carlitz, "The sum of the first $n$ terms of an ${ }_{5} F_{4}$," Boll. Un. Mat. Ital., (3), v. 19, 1965 , pp. 436-440. MR 30 * 4993 .

2. T. M. MACROBERT \& F. M. RAGAB, " $E$-function series whose sums are constants." Math. Z., v. 78, 1962, pp. 231-233. MR $27 * 354$. 1966.

3. L. J. Slater, Generalised Hypergeometric Functions, Cambridge Univ. Press, London,

4. A. Verma, "A class of expansions of $G$-functions and the Laplace transform," Math. Comp., v. 15, 1965, pp. 661-664.

\section{The Fast Fourier Transform Recursive Equations for Arbitrary Length Records}

\section{By G. D. Bergland}

1. Introduction. In Cooley and Tukey's paper on the Fast Fourier Transform [1], a procedure was suggested which allows operation on a time series of length $N$, where $N$ is the product of an arbitrary number of integers greater than 1 . An alternate procedure is reported in this paper which has the advantage of leading directly to a set of recursive equations for this general case. The notation and methods used have been patterned as much as possible to those of Cooley and Tukey so that the reader can go directly from their paper to this one. These results were noted and used by the author in his Ph.D. dissertation [2].

2. The Recursive Equations when $N=r_{1} r_{2} \cdots r_{m}$. Consider the problem of evaluating a complex Fourier series of the form

$$
X(j)=\sum_{k=0}^{N-1} A(k) W^{j k}
$$

where $W=e^{2 \pi i / N}, j=0,1,2, \cdots, N-1$.

First $j$ and $k$ must be expressed in the following variable radix representation.

Received August 24, 1966. 